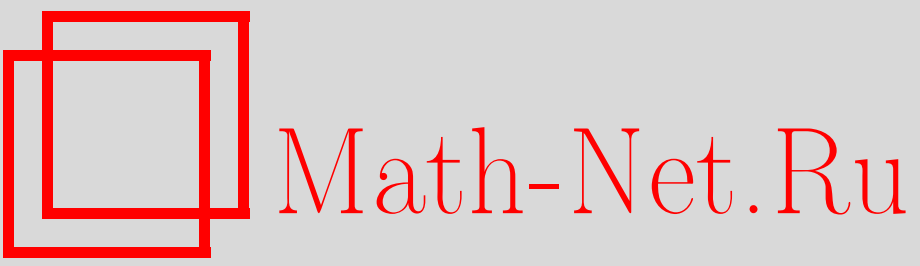

С. В. Лотоцкий, Б. Л. Розовский, Пассивное скалярное уравнение в турбулентном несжимаемом гауссовом поле скоростей, УМH, 2004, том 59, выпуск 2, 105120

DOI: https://doi.org/10.4213/rm719

Использование Общероссийского математического портала Math-Net.Ru подразумевает, что вы прочитали и согласны с пользовательским соглашением http://www . mathnet.ru/rus/agreement

Параметры загрузки:

IP : 34.229 .108 .108

26 апреля 2023 г., $15: 10: 00$ 


\title{
ПАССИВНОЕ СКАЛЯРНОЕ УРАВНЕНИЕ В ТУРБУЛЕНТНОМ НЕСЖИМАЕМОМ ГАУССОВОМ ПОЛЕ СКОРОСТЕЙ
}

\author{
С.В. Лотоцкий, Б. Л. Розовский
}

\begin{abstract}
Рассматривается эволюция во времени пассивного скаляра в турбулентном однородном несжимаемом гауссовом потоке. Турбулентная природа потока приводит к негладким коэффициентам соответствующего эволюционного уравнения. С помощью разложения винеровского хаоса строится сильное (в вероятностном смысле) решение уравнения и изучаются свойства этого решения. В числе полученных результатов - определенная $L_{p}$-регулярность решения и представляющая формула типа Фейнмана-Каца (или лагранжева). Резултаты применимы и к вязким, и к консервативньп потокам.
\end{abstract}

Библиография: 14 названий

\section{СОДЕРЖАНИЕ}

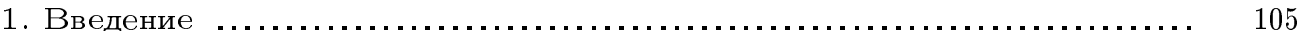

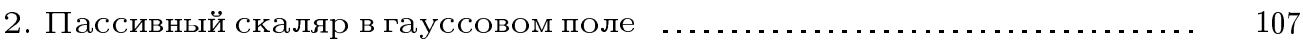

3. Обзор винеровского хаоса ...................................... 109

4. Решение уравнения пассивного скаляра с помощью винеровского ха-

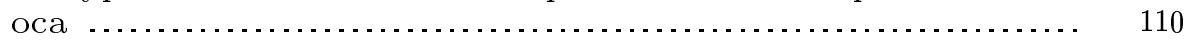

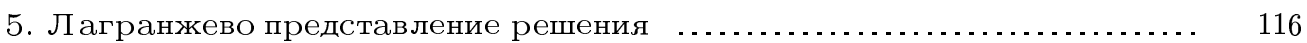

Список литературы ..................................................... 119

\section{1. Введение}

Если $\mathbf{v}=\mathbf{v}(t, x)$ - гладкое векторное поле в $\mathbb{R}^{d}$, то существует единственное классическое решение $\theta=\theta(t, x)$ невязкого уравнения переноса

$$
\frac{\partial \theta}{\partial t}+\mathbf{v} \cdot \nabla \theta=0, \quad t>0, \quad \theta(0, x)=\theta_{0}(x) .
$$

Это решение можно представить в виде

$$
\theta(t, x)=\theta_{0}\left(X_{t, 0}^{x}\right)
$$

Работа первого автора была частично поддержана Sloan Research Fellowship, NSF CAREER award DMS-0237724 и ARO Grant DAAD19-02-1-0374; работа второго автора была частично поддержана ONR Grant N0014-03-1-0027 и ARO Grant DAAD19-02-1-0374. 
где $X=X_{s, t}^{x}-$ поток, порожденньй векторным полем v:

$$
\frac{d X_{s, t}^{x}}{d t}=\mathbf{v}\left(t, X_{s, t}^{x}\right), \quad t>s, \quad X_{s, s}^{x}=x
$$

Если поле скоростей $\mathbf{v}$ не является гладким как функция $x$, то, вообще говоря, для уравнения (1.1) нет теорем существования, в то время как уравнение (1.3) может иметь более одного решения. Следовательно, связь между уравнениями (1.1) и (1.3) становится неясной, и представление решения в виде (1.2) сомнительно.

Для негладких векторных полей $\mathbf{v}$ анализ любого из уравнений (1.1) и (1.3) становится невозможньм без дальнейшей спецификации функции v. В [11] показано, что во многих физических моделях, таких, как турбулентные потоки и т. д., функцию $\mathbf{v}=$ $\left(v^{1}(t, x), \ldots, v^{d}(t, x)\right)$ можно представить в виде

$$
v^{i}(t, x)=\sum_{k \geqslant 1} \sigma_{k}^{i}(x) \dot{w}_{k}(t)
$$

где каждая функция $\sigma_{k}^{i}(x)$ удовлетворяет условию Гёльдера по $x$ с показателем, меньшим единицы, причем имеет место равенство $\sum_{k \geqslant 1} \sigma_{k}^{i}(x) \sigma_{k}^{j}(y)=C^{i j}(x-y)$ для некоторых функций $C^{i j}, \mathrm{a}\left(w_{k}, k \geqslant 1\right)$ - независимые стандартные броуновские движения на некотором вероятностном пространстве $(\Omega, \mathscr{F}, \mathrm{P})$. Тогда уравнение $(1.3)$ становится стохастическим дифференшиальньм уравнением

$$
d\left(X_{s, t}^{x}\right)^{i}=\sigma_{k}^{i}\left(X_{s, t}^{x}\right) d w_{k}(t), \quad t>s, \quad\left(X_{s, s}^{x}\right)^{i}=x^{i}
$$

Вообще говоря, это уравнение не имеет сильного решения, т.е. функция $X_{s, t}^{x}$, вообше говоря, не является измеримой относительно сигма-алгебры $\mathscr{F}_{s, t}^{W}$, порожденной прирашениями $w_{k}(v)-w_{k}(u), s \leqslant u<v \leqslant t, k \geqslant 1$. С другой стороны, стандартный факт состоит в том, что, при перечисленных выше предположениях относительно $\sigma_{k}^{i}$, уравнение (1.5) всегда имеет единственное слабое решение (см., например, [1]).

В [11] авторы предприняли систематическое изучение семейства операторов

$$
S_{s, t}: f(x) \mapsto \mathrm{E}\left(f\left(X_{s, t}^{x}\right) \mid \mathscr{F}_{s, t}^{W}\right)
$$

для подходяших функций $f$, где $X$ - слабое решение (1.5). Если функции $\sigma_{k}^{i}$ липшицевы, то функция $X_{s, t}^{x}$ является $\mathscr{F}_{s, t}^{W}$-измеримой. Кроме того, в [13] показано, что в этом случае функция $\theta(t, x)=\theta_{0}\left(X_{t, 0}^{x}\right)=S_{t, 0} \theta_{0}(x)$ является единственньм обобщенным решением стохастического дифференциального уравнения Стратоновича (с частными производньми)

$$
d \theta+\sum_{i, k} \sigma_{k}^{i} \frac{\partial \theta}{\partial x^{i}} \circ d w_{k}, \quad t>0, \quad \theta(0, x)=\theta_{0}(x) .
$$

Если функции $\sigma_{k}^{i}$ не являются липшицевыми, то связь между операторами $S_{s, t}$ и уравнением (1.7) становится неясной. В частности, вообше говоря, уравнение (1.7) не имеет решения в традищионном смысле, ни в классическом, ни в обобшенном, и даже если 
определить решение формулой $S_{t, 0} \theta_{0}(x)$ (ср. с [5]), неясно, в каком смысле будет выполняться уравнение. Наконец, никакие теоремы существования не указьвают пути вычисления условного ожидания в (1.6) для данного конкретного векторного поля $\mathbf{v}$.

Цель настоящей статьи - показать, что если векторное поле $\mathbf{v}$ не является гладким, но по-прежнему бездивергентно в обобшенном смысле и если $\theta_{0} \in L_{p}\left(\mathbb{R}^{d}\right), 2 \leqslant p \leqslant \infty$, то уравнение (1.7) имеет единственное сильное обобщенное решение. Более точно, показано, что существует случайное векторное поле $\theta=\theta(t, x)$, удовлетворяющее следуюшим условиям для любого $t>0$ :

(i) $\theta(t, \cdot) \in L_{p}\left(\Omega \times \mathbb{R}^{d}\right), 2 \leqslant p \leqslant \infty$,

(ii) $\theta(t, \cdot)$ является $\mathscr{F}_{0, t}^{W}$-измеримой,

(iii) для любой гладкой функции $\varphi$ с компактньм носителем соотношение

$$
(\theta, \varphi)(t)=\left(\theta_{0}, \varphi\right)+\sum_{i, k} \int_{0}^{t}\left(\theta, \sigma_{k}^{i} \frac{\partial \varphi}{\partial x^{i}}\right)(s) \circ d w_{k}(s)
$$

вьполняется с вероятностью 1 , где $(\cdot, \cdot)$ означает скалярное произведение в $\mathbb{R}^{d}$.

Это случайное поле допускает лагранжево представление

$$
\theta(t, x)=\mathrm{E}\left(\theta_{0}\left(X_{t, x}(0)\right) \mid \mathscr{F}_{t}^{W}\right) \quad(\text { Р-п.н. })
$$

где

$$
X_{t, x}(s)=x+\int_{s}^{t} \sigma\left(X_{t, x}(r)\right) \overleftarrow{d W}(r)+\sqrt{2 \nu}(\widetilde{W}(t)-\widetilde{W}(s))
$$

здесь $\widetilde{W}$ - стандартное броуновское движение, независимое от $W$. Решение уравнения (1.8), как и его моменты, можно вычислить с помощью разложения винеровского хаоса (см. также замечание 5.6).

\section{2. Пассивный скаляр в гауссовом поле}

Рассмотрим следующее уравнение переноса для описания эволющии пассивного скаляра $\theta$ в случайном поле скоростей $\mathbf{v}$

$$
\dot{\theta}(t, x)=\frac{1}{2} \nu \Delta \theta(t, x)-\mathbf{v}(t, x) \cdot \nabla \theta(t, x)+f(t, x) ; \quad x \in \mathbb{R}^{d}, \quad d>1 .
$$

В (2.1) символы $\Delta$ и $\nabla$ означают соответственно оператор Лапласа и градиент. Наш интерес к этому уравнению вызван продолжаюшимися успехами в изучении турбулентной задачи переноса (см. [5]-[7], [10] и т. д.)

Предположим, что в (2.1) векторное поле $\mathbf{v}=\mathbf{v}(t, x) \in \mathbb{R}^{d}, d \geqslant 2$, является изотропным и гауссовьм, с нулевым средним и с ковариацией $\mathrm{E}\left(v^{i}(t, x) v^{j}(s, y)\right)=$ $\delta(t-s) C^{i j}(x-y)$, где $C=\left(C^{i j}(x), i, j=1, \ldots, d\right)$ - матричнозначная функция. Хорошо известно (см., например, [11]), что в физически интересных моделях, таких, например, как скорость Крайчнана [10], матричнозначная функция $C=C(x)$ имеет преобразование Фурье $\widehat{C}=\widehat{C}(z)$, равное

$$
\widehat{C}(z)=\frac{A_{0}}{\left(1+|z|^{2}\right)^{(d+\alpha) / 2}}\left(a \frac{z z^{*}}{|z|^{2}}+\frac{b}{d-1}\left(I-\frac{z z^{T}}{|z|^{2}}\right)\right),
$$


где $z^{*}$ - вектор-строка $\left(z_{1}, \ldots, z_{d}\right), z$ - соответствующий вектор-столбец, $|z|^{2}=z^{*} z$, $I$ - единичная матрица, $\alpha>0, a \geqslant 0, b \geqslant 0, A_{0}>0$-вешественные числа. Как и в [11], предположим, что $0<\alpha<2$.

Прямое вычисление показывает (ср. с [2]), что векторное поле $\mathbf{v}=\left(v^{1}, \ldots, v^{d}\right)$ может быть записано в виде

$$
v^{i}(t, x)=\sum_{k \geqslant 0} \sigma_{k}^{i}(x) \dot{w}_{k}(t)
$$

где $\dot{w}_{k}(t), k \geqslant 1,-$ независимые стандартные гауссовы белые шумы и $\left\{\sigma_{k}, k \geqslant 1\right\}-$ полная ортонормированная система в пространстве $H_{C}$ (гильбертово пространство с воспроизводяшим ядром, соответствуюшим функщии-ядру $C$ ). Пространство $H_{C}$ есть соболевское пространство $H_{2}^{(d+\alpha) / 2}\left(\mathbb{R}^{d} ; \mathbb{R}^{d}\right)$ или его часть. Из (2.3) следует, что $\sum_{k} \sigma_{k}^{i}(x) \sigma_{k}^{j}(y)=C^{i j}(x-y)$ для всех $x, y$; в частности, $\sigma_{k}^{i}(x) \sigma_{k}^{j}(x)=C^{i j}(0)$ для всех $x$.

Если $a>0$ и $b>0$, то матрица $\widehat{C}$ обратима и

$$
H_{C}=\left\{f \in \mathbb{R}^{d}: \int_{\mathbb{R}^{d}} \widehat{f}^{*}(z) \widehat{C}^{-1}(z) \widehat{f}(z) d z<\infty\right\}=H_{2}^{(d+\alpha) / 2}\left(\mathbb{R}^{d} ; \mathbb{R}^{d}\right)
$$

поскольку $\|\widehat{C}(z)\| \sim\left(1+|z|^{2}\right)^{-(d+\alpha) / 2}$.

Если $a>0$ и $b=0$, то

$$
H_{C}=\left\{f \in \mathbb{R}^{d}: \int_{\mathbb{R}^{d}}|\widehat{f}(z)|^{2}\left(1+|z|^{2}\right)^{(d+\alpha) / 2} d z<\infty ; z z^{*} \widehat{f}(z)=|z|^{2} \widehat{f}(z)\right\}
$$

есть подмножество градиентных полей в $H_{2}^{(d+\alpha) / 2}\left(\mathbb{R}^{d} ; \mathbb{R}^{d}\right)$, т.е. семейство таких векторных полей $f$, что $\widehat{f}(z)=z \widehat{F}(z)$ для некоторой скалярной функции $F \in$ $H_{2}^{(d+\alpha+1) / 2}\left(\mathbb{R}^{d}\right)$.

Если $a=0$ и $b>0$, то

$$
H_{C}=\left\{f \in \mathbb{R}^{d}: \int_{\mathbb{R}^{d}}|\widehat{f}(z)|^{2}\left(1+|z|^{2}\right)^{(d+\alpha) / 2} d z<\infty ; z^{*} \widehat{f}(z)=0\right\}
$$

есть подмножество бездивергентных полей в $H_{2}^{(d+\alpha) / 2}\left(\mathbb{R}^{d} ; \mathbb{R}^{d}\right)$.

По теоремам вложения, каждая функция $\sigma_{k}^{i}$ ограничена и непрерывна на $\mathbb{R}^{d}$; действительно, каждая функция $\sigma_{k}^{i}$ удовлетворяет условию Гёльдера порядка $\alpha / 2$. Кроме того, будучи элементом соответствующего пространства $H_{C}$, каждая функция $\sigma_{k}$ является градиентным полем при $b=0$ и бездивергентна при $a=0$.

Чтобы упростить дальнейшее изложение и сделать модель (2.1) более значимой физически, мы рассмотрим бездивергентное векторное поле и предположим, что исходный стохастический дифференциал в (2.3) есть дифференциал в смысле Стратоновича. В этих предположениях уравнение (2.1) принимает вид

$$
d \theta(t, x)=\frac{1}{2} \nu \Delta \theta(t, x) d t-\sum_{k} \sigma_{k}(x) \cdot \nabla \theta(t, x) \circ d w_{k}(t) .
$$

Если функции $\sigma_{k}$ бездивергентны, то есть эквивалентная формулировка Ито:

$$
d \theta(t, x)=\frac{1}{2}\left(\nu \Delta \theta(t, x)+C^{i j}(0) D_{i} D_{j} \theta(t, x)\right) d t-\sigma_{k}^{i}(x) D_{i} \theta(t, x) d w_{k}(t),
$$

где $D_{i}=\partial / \partial x^{i}$ и проводится суммирование по повторяющимся индексам. 
Мы будем изучать уравнение (2.5) в следующих предположениях.

A1. Существует фиксированньй стохастический базис $\mathbb{F}=\left(\Omega, \mathscr{F},\left\{\mathscr{F}_{t}\right\}_{t \geqslant 0}, \mathrm{P}\right)$, удовлетворяющий обычным условиям, и $\left(w_{k}(t), k \geqslant 1, t \geqslant 0\right)$ - семейство независимых стандартных винеровских процессов на $\mathbb{F}$.

A2. Для любого $k$ векторное поле $\sigma_{k}$ является бездивергентным элементом соболевского пространства $H_{2}^{(d+\alpha) / 2}\left(\mathbb{R}^{d} ; \mathbb{R}^{d}\right), 0<\alpha<2, d \geqslant 2$.

A3. Для любых $x, y$ в $\mathbb{R}^{d}$ имеем $\sum_{k} \sigma_{k}^{i}(x) \sigma_{k}^{j}(y)=C^{i j}(x-y)$, и матричнозначная функция $C=C(x)$ удовлетворяет $(2.2)$.

A4. Начальное условие $\theta_{0}$ неслучайно и принадлежит $L_{2}\left(\mathbb{R}^{d}\right) ; \nu \geqslant 0$ - вещественное число.

Наша цель - установить существование, единственность и свойства регулярности решения уравнения (2.5), используя винеровский хаос.

\section{3. Обзор винеровского хаоса}

Пусть $\mathbb{F}=\left(\Omega, \mathscr{F},\left\{\mathscr{F}_{t}\right\}_{t \geqslant 0}, \mathrm{P}\right)$ - стохастический базис, удовлетворяюший обычным условиям. Рассмотрим на $\mathbb{F}$ семейство $\left(w_{k}(t), k \geqslant 1, t \geqslant 0\right)$ независимых стандартных винеровских процессов. При фиксированном $0<T<\infty$ обозначим через $\mathscr{F}_{T}^{W}$ сигма-алгебру, порожденную $w_{k}(t), k \geqslant 1,0<t<T$, и обозначим через $L_{2}\left(\mathscr{F}_{T}^{W}\right)$ семейство $\mathscr{F} T$-измеримых квадратично интегрируемых случайных величин.

Фиксируем Фурье-базис из косинусов $\left\{m_{k}, k \geqslant 1\right\}$ в $L_{2}((0, T))$ с

$$
m_{1}(t)=\frac{1}{\sqrt{T}}, \quad m_{k}(t)=\sqrt{\frac{2}{T}} \cos \left(\frac{\pi(k-1) t}{T}\right), \quad k \geqslant 2 .
$$

Рассмотрим семейство мультииндексов

$$
\mathscr{J}=\left\{\alpha=\left(\alpha_{i}^{k}, i, k \geqslant 1\right), \alpha_{i}^{k} \in\{0,1,2, \ldots\}, \sum_{i, k} \alpha_{i}^{k}<\infty\right\} .
$$

Множество $\mathscr{J}$ счетно, и для любого $\alpha \in \mathscr{J}$ лишш конечное число индексов $\alpha_{i}^{k}$ отлично от нуля. При $\alpha \in \mathscr{J}$ положим $|\alpha|=\sum_{i, k} \alpha_{i}^{k}, \alpha !=\prod_{i, k} \alpha_{i}^{k} !$ и

$$
\xi_{\alpha}=\frac{1}{\sqrt{\alpha !}} \prod_{i, k} H_{\alpha_{i}^{k}}\left(\xi_{i k}\right), \quad \text { где } \quad \xi_{i k}=\int_{0}^{T} m_{i}(s) d w_{k}(s)
$$

и

$$
H_{n}(t)=e^{t^{2} / 2} \frac{d^{n}}{d t^{n}} e^{-t^{2} / 2}
$$

- $n$-й многочлен Эрмита. В частности, если $\alpha \in \mathscr{J}$ удовлетворяет условию $\alpha_{i}^{k}=1$ при $i=j$ и $k=l$ и $\alpha_{i}^{k}=0$ в других случаях, то $\xi_{\alpha}=\xi_{j l}$.

ОПРЕДЕЛЕНИЕ 3.1. Пространство $L_{2}(\mathscr{F} T)$ называется пространством винеровского хаоса, а $N$-м подпространством винеровского хаоса называется подпространство $L_{2}\left(\mathscr{F}_{T}^{W}\right)$, порожденное элементами $\xi_{\alpha} \mathrm{c}|\alpha|=N$.

Следуюшая теорема - классический результат Камерона и Мартина [4]. 
ТЕОРема 3.2. Набор $\left\{\xi_{\alpha}, \alpha \in \mathscr{J}\right\}$ является ортонормированным базисом в пространстве $L_{2}\left(\mathscr{F}_{T}^{W}\right)$.

Кроме исходного источника [4], доказательство этой теоремы можно найти и во многих других местах, например, в [8]. По теореме 3.2 , каждый элемент $v$ пространства $L_{2}\left(\mathscr{F}_{T}^{W}\right)$ можно представить в виде $v=\sum_{\alpha \in \mathscr{J}} v_{\alpha} \xi_{\alpha}$, где $v_{\alpha}=\mathrm{E}\left(v \xi_{\alpha}\right)$.

\section{4. Решение уравнения пассивного скаляра с помощью винеровского хаоса}

Продолжая использовать соглашение о суммировании, определим операторы $\mathscr{A}=$ $\frac{1}{2}\left(\nu \Delta+C^{i j}(0) D_{i} D_{j}\right)$ и $\mathscr{M}_{k}=\sigma_{k}^{i}(x) D_{i}$. Тогда уравнение $(2.5)$ принимает вид

$$
\theta(t, x)=\theta_{0}(x)+\int_{0}^{t} \mathscr{A} \theta(s, x) d s+\int_{0}^{t} \mathscr{M}_{k} \theta(s, x) d w_{k}(s) d s
$$

В этом разделе, начиная отсюда, мы не будем явно указывать зависимость различных функций от $x$.

Заметим, что для любой функции $f \in H_{2}^{1}\left(\mathbb{R}^{d}\right)$ имеем

$$
\sum_{k \geqslant 1}\left\|\mathscr{M}_{k} f\right\|_{L_{2}\left(\mathbb{R}^{d}\right)}^{2}=\left(\sigma_{k}^{j} \sigma_{k}^{i} D_{i} f, D_{j} f\right)=\left(C^{i j}(0) D_{i} f, D_{j} f\right)
$$

где $(\cdot, \cdot)$ - скалярное произведение в $L_{2}\left(\mathbb{R}^{d}\right)$. Так как матрица $C(0)$ положительно определена, то сушествуют такие положительные числа $c_{1}$ и $c_{2}$, что для любой функции $f \in H_{2}^{1}\left(\mathbb{R}^{d}\right)$ вьполняется соотношение

$$
c_{1}\|\nabla f\|_{L_{2}\left(\mathbb{R}^{d}\right)}^{2} \leqslant \sum_{k \geqslant 1}\left\|\mathscr{M}_{k} f\right\|_{L_{2}\left(\mathbb{R}^{d}\right)}^{2} \leqslant c_{2}\|\nabla f\|_{L_{2}\left(\mathbb{R}^{d}\right)}^{2}
$$

Равенство (4.1) показьвает также, что уравнение (2.5) есть стохастическое параболическое уравнение [14]; оно является суперпараболическим при $\nu>0$ и вполне вырожденньм при $\nu=0$.

При $\alpha \in \mathscr{J}$ определим функщии $\theta_{\alpha}$ следуюшим образом:

$$
\theta_{\alpha}(t)=\theta_{0} I(|\alpha|=0)+\int_{0}^{t} \mathscr{A} \theta_{\alpha}(s) d s+\int_{0}^{t} \sum_{i, k} \sqrt{\alpha_{i}^{k}} \mathscr{M}_{k} \theta_{\alpha^{-}(i, k)}(s) m_{i}(s) d s
$$

где $\alpha^{-}(i, k)$ - мультииндекс с компонентами

$$
\left(\alpha^{-}(i, k)\right)_{j}^{l}= \begin{cases}\max \left(\alpha_{i}^{k}-1,0\right), & \text { если } i=j \text { и } k=l, \\ \alpha_{j}^{l} & \text { в противном случае. }\end{cases}
$$

ЛЕмма 4.1. В предположсениях А2-A4 система уравнений (4.3) имеет единственное решение, в котором $\theta_{\alpha}$ является гладкой ограниченной функиией $x$ при 
$t>0$, и если $T_{t}, t \geqslant 0,-$ полугруппа теплопроводности, порожсденная оператором $\mathscr{A}$, то при любом $N \geqslant 0$ имеем

$$
\begin{aligned}
& \sum_{|\alpha|=N}\left|\theta_{\alpha}(t, x)\right|^{2} \\
& \quad=\sum_{k_{1}, \ldots, k_{N}=1}^{\infty} \int_{0}^{t} \int_{0}^{s_{N}} \cdots \int_{0}^{s_{2}}\left|T_{t-s_{N}} \mathscr{M}_{k_{N}} \cdots T_{s_{2}-s_{1}} \mathscr{M}_{k_{1}} T_{s_{1}} \theta_{0}(x)\right|^{2} d s^{N}
\end{aligned}
$$

əде $d s^{N}=d s_{1} \cdots d s_{N} u$

$$
\begin{aligned}
& \sum_{|\alpha|=N}\left|\nabla \theta_{\alpha}(t, x)\right|^{2} \\
& \quad=\sum_{k_{1}, \ldots, k_{N}=1}^{\infty} \int_{0}^{t} \int_{0}^{s_{N}} \cdots \int_{0}^{s_{2}}\left|\nabla T_{t-s_{N}} \mathscr{M}_{k_{N}} \cdots T_{s_{2}-s_{1}} \mathscr{M}_{k_{1}} T_{s_{1}} \theta_{0}(x)\right|^{2} d s^{N},
\end{aligned}
$$

əəe $d s^{N}=d s_{1} \cdots d s_{N}$.

ДокАЗАТЕЛЬСтво. Эти результаты непосредственно следуют из (4.3) индукцией по $|\alpha|$; подробности можно найти в $[12 ;$ предложение A.1].

Теорема 4.2. B предположениях A1-A4 фиксируем $T>0$ и при $\alpha \in \mathscr{J}$ определим $\theta_{\alpha}(t)$ и $\xi_{\alpha}$ формулами (4.3) и (3.2) соответственно. Тогда справедливь следующие утверэмдения.

1. При любом $\nu \geqslant 0$ и любом $t \in[0, T]$ ряд

$$
\sum_{\alpha \in \mathscr{J}} \theta_{\alpha}(t) \xi_{\alpha}
$$

сходится в $L_{2}\left(\Omega ; L_{2}\left(\mathbb{R}^{d}\right)\right)$ к некоторому прочессу $\theta=\theta(t)$.

2. Если $\nu>0$, то при любом $\varphi \in C_{0}^{\infty}\left(\mathbb{R}^{d}\right)$ прочесс $\theta$ удовлетворяет соотношению

$$
\begin{aligned}
(\theta, \varphi)(t)=\left(\theta_{0}, \varphi\right) & -\frac{1}{2} \nu \int_{0}^{t}(\nabla \theta, \nabla \varphi)(s) d s \\
& -\frac{1}{2} \int_{0}^{t} C^{i j}(0)\left(D_{i} \theta, D_{j} \varphi\right)(s) d s-\int_{0}^{t}\left(\sigma_{k}^{i} D_{i} \theta, \varphi\right) d w_{k}(s)
\end{aligned}
$$

с вероятностью 1 для всех $t \in[0, T]$ одновременно, где $(\cdot, \cdot)$ - скалярное произведение в $L_{2}\left(\mathbb{R}^{d}\right)$. Кроме того,

$$
\mathrm{E}\|\theta\|_{L_{2}\left(\mathbb{R}^{d}\right)}^{2}(t)+\nu \int_{0}^{t} \mathrm{E}\|\nabla \theta\|_{L_{2}\left(\mathbb{R}^{d}\right)}^{2}(s) d s=\left\|\theta_{0}\right\|_{L_{2}\left(\mathbb{R}^{d}\right)}^{2} .
$$

3. Если $\nu=0$, то для любого $\varphi \in C_{0}^{\infty}\left(\mathbb{R}^{d}\right)$ процесс $\theta$ удовлетворяет соотношению

$$
(\theta, \varphi)(t)=\left(\theta_{0}, \varphi\right)+\frac{1}{2} \int_{0}^{t} C^{i j}(0)\left(\theta, D_{i} D_{j} \varphi\right)(s) d s+\int_{0}^{t}\left(\theta, \sigma_{k}^{i} D_{i} \varphi\right) d w_{k}(s)
$$

с вероятностью 1 для всех $t \in[0, T]$ одновременно. Кроме того,

$$
\mathrm{E}\|\theta\|_{L_{2}\left(\mathbb{R}^{d}\right)}^{2}(t) \leqslant\left\|\theta_{0}\right\|_{L_{2}\left(\mathbb{R}^{d}\right)}^{2}
$$


ЗАмечание 4.3. Равенства (4.7) и (4.9) означают, что $\theta=\theta(t, x)$ является решением уравнения переноса (2.5) в традиционном смысле теории стохастических дифференциальных уравнений с частньми производными, т.е. это - сильное решение в стохастическом смысле, удовлетворяющее соответствующему уравнению в обобщенном смысле. Это решение также единственно в классе $L_{2}\left((0, T) \times \Omega ; L_{2}\left(\mathbb{R}^{d}\right)\right)$ случайных функций, поскольку любое другое решение будет иметь то же разложение винеровского хаоса. Не входя в подробности, заметим, что на самом деле можно установить единственность в гораздо более широком классе обобшенных случайных функций.

Доказательство теоремы 4.2 проводится в три шага: (1) устанавливаются сходимость ряда (4.6) и соответствуюшие энергетические оценки; (2) устанавливается предсказуемость $\theta ;(3)$ устанавливаются равенства (4.7) и (4.9).

Сходимость ряда (4.6) и соответствуюшие энергетические оценки (шаг 1) вытекают из двух следующих лемм.

Лемма 4.4. Предположим, что $\nu \geqslant 0$. Введем

$$
\theta_{N}(t, x)=\sum_{n=0}^{N} \sum_{|\alpha|=n} \theta_{\alpha}(t, x) \xi_{\alpha} .
$$

Тогда для любого $t \in[0, T]$ имеем

$$
\begin{aligned}
& \mathrm{E}\left\|\theta_{N}\right\|_{L_{2}\left(\mathbb{R}^{d}\right)}^{2}(t)=\left\|\theta_{0}\right\|_{L_{2}\left(\mathbb{R}^{d}\right)}^{2}-\nu \sum_{n=0}^{N} \sum_{|\alpha|=n} \int_{0}^{t}\left\|\nabla \theta_{\alpha}\right\|_{L_{2}\left(\mathbb{R}^{d}\right)}^{2}(s) d s \\
& -\sum_{k_{1}, \ldots, k_{N+1}=1}^{\infty} \int_{0}^{t} \cdots \int_{0}^{s_{2}}\left\|\mathscr{M}_{k_{N+1}} T_{s-s_{N}} \mathscr{M}_{k_{N}} \cdots T_{s_{2}-s_{1}} \mathscr{M}_{k_{1}} T_{s_{1}} \theta_{0}\right\|_{L_{2}\left(\mathbb{R}^{d}\right)}^{2} d s^{N} d s .
\end{aligned}
$$

ДокАЗАТЕЛЬСтво. По лемме 4.1, после интегрирования по $x$ получаем

$$
\begin{aligned}
& \sum_{|\alpha|=N}\left\|\theta_{\alpha}\right\|_{L_{2}\left(\mathbb{R}^{d}\right)}^{2}(t) \\
& \quad=\sum_{k_{1}, \ldots, k_{N}=1}^{\infty} \int_{0}^{t} \int_{0}^{s_{N}} \cdots \int_{0}^{s_{2}}\left\|T_{t-s_{N}} \mathscr{M}_{k_{N}} \ldots T_{s_{2}-s_{1}} \mathscr{M}_{k_{1}} T_{s_{1}} \theta_{0}\right\|_{L_{2}\left(\mathbb{R}^{d}\right)}^{2} d s^{N}
\end{aligned}
$$

Если $F_{N}(t)=\sum_{|\alpha|=N}\left\|\theta_{\alpha}(t)\right\|_{L_{2}(\mathbb{R})}^{2}$, то

$$
\begin{aligned}
& \frac{d}{d t} F_{N}(t) \\
& =\sum_{k_{1}, \ldots, k_{N}=1}^{\infty} \int_{0}^{t} \int_{0}^{s_{N-1}} \cdots \int_{0}^{s_{2}}\left\|\mathscr{M}_{k_{N}} T_{t-s_{N-1}} \mathscr{M}_{k_{N-1}} \cdots T_{s_{2}-s_{1}} \mathscr{M}_{k_{1}} T_{s_{1}} \theta_{0}\right\|_{L_{2}(\mathbb{R})}^{2} d s^{N-1} \\
& +\underset{k_{1}, \ldots, k_{N}=1}{2} \int_{0}^{t} \cdots \int_{0}^{s_{2}}\left(\mathscr{A} T_{t-s_{N}} \mathscr{M}_{k_{N}} \cdots T_{s_{1}} \theta_{0}, T_{t-s_{N}} \mathscr{M}_{k_{N}} \cdots T_{s_{2}-s_{1}} \mathscr{M}_{k_{1}} T_{s_{1}} \theta_{0}\right) d s^{N} .
\end{aligned}
$$


Остается заметить, что для любой гладкой функции $f=f(x)$ имеем

$$
2(\mathscr{A} f, f)=-\nu\|\nabla f\|_{L_{2}(\mathbb{R})}^{2}-\sum_{k \geqslant 1}\left\|\mathscr{M}_{k} f\right\|_{L_{2}(\mathbb{R})}^{2} .
$$

Отсюда следует равенство (4.11).

Заметим, что из $(4.11)$ следует и сходимость в $L_{2}\left(\Omega ; L_{2}\left(\mathbb{R}^{d}\right)\right)$ ряда $\sum_{\alpha} \theta_{\alpha}(t) \xi_{\alpha}$ для любого $t \in[0, T]$, и неравенство (4.10).

ЛЕмма 4.5. Если $\nu>0$, то для любого $t \in[0, T]$ имеем

$$
\lim _{N \rightarrow \infty} \sum_{k_{1}, \ldots, k_{N+1}=1}^{\infty} \int_{0}^{t} \cdots \int_{0}^{s_{2}}\left\|\mathscr{M}_{k_{N+1}} T_{s-s_{N}} \mathscr{M}_{k_{N}} \cdots T_{s_{2}-s_{1}} \mathscr{M}_{k_{1}} T_{s_{1}} \theta_{0}\right\|_{L_{2}\left(\mathbb{R}^{d}\right)}^{2} d s^{N} d s=0
$$

ДоКАЗАТЕЛЬСТВо. Положим

$$
F_{N}(t)=\sum_{k_{1}, \ldots, k_{N+1}=1}^{\infty} \int_{0}^{t} \cdots \int_{0}^{s_{2}}\left\|\mathscr{M}_{k_{N+1}} T_{s-s_{N}} \mathscr{M}_{k_{N}} \cdots T_{s_{2}-s_{1}} \mathscr{M}_{k_{1}} T_{s_{1}} \theta_{0}\right\|_{L_{2}\left(\mathbb{R}^{d}\right)}^{2} d s^{N} d s .
$$

Согласно (4.2) и лемме 4.1 имеем

$$
F_{N}(t) \leqslant c_{2} \sum_{|\alpha|=N} \int_{0}^{t}\left\|\nabla \theta_{\alpha}\right\|_{L_{2}\left(\mathbb{R}^{d}\right)}^{2}(s) d s
$$

Тогда из леммы 4.4 следует, что ряд $\sum_{N \geqslant 0} F_{N}(t)$ сходится для всех $t \in[0, T]$. Следовательно, $\lim _{N \rightarrow \infty} F_{N}(t)=0$, откуда следует утверждение леммы.

Заметим, что из (4.11) и (4.14) следует формула (4.8).

ЗАмечАниЕ 4.6. Анализ приведенных выше доказательств показывает, что заключения лемм 4.1, 4.4 и 4.5 не зависят от выбора базиса $\left\{m_{i}, i \geqslant 1\right\}$ в $L_{2}((0, T))$. Иначе говоря, если $\left\{m_{i}, i \geqslant 1\right\}$ - некоторьй ортонормированньй базис в $L_{2}((0, T))$ и процесс $\theta$ определен формулой (4.6), то для любого $t \in[0, T]$ величина $\theta(t)$ принадлежит $L_{2}\left(\Omega ; L_{2}\left(\mathbb{R}^{d}\right)\right)$, удовлетворяет соответствуюшей энергетической оценке и $\mathscr{F}_{T}^{W}$-измерима.

Следуюшая лемма показьвает, что конструкция процесса $\theta$ не зависит от выбора базиса $\left\{m_{i}, i \geqslant 1\right\}$ в $L_{2}((0, T))$. Эта лемма является также ключом к установлению предсказуемости процесса $\theta$.

Лемма 4.7. Пусть $\theta$ - процесс, определенный формулой (4.6), и пусть $\bar{m}_{i}(t), i \geqslant 1$, - другой ортонормированньй базис в $L_{2}((0, T))$. Тогда $\theta(t)=$ $\sum_{\alpha \in \mathscr{J}} \bar{\theta}_{\alpha}(t) \bar{\xi}_{\alpha}$, əдe

$$
\bar{\xi}_{\alpha}=\frac{1}{\sqrt{\alpha !}} \prod_{i, k} H_{\alpha_{i}^{k}}\left(\bar{\xi}_{i k}\right), \quad \bar{\xi}_{i k}=\int_{0}^{T} \bar{m}_{i}(s) d w_{k}(s)
$$

икоэффициенты $\bar{\theta}_{\alpha}(t)$ удовлетворяют системе уравнений (4.3) с $\bar{m}_{i}$ вместо $m_{i}$. 
ДокАЗАТЕЛЬСТвО. Пусть $h=\left(h_{1}(t), \ldots, h_{N}(t)\right)$ - конечньй набор ограниченных измеримых функций на $(0, T)$. Введем величину

$$
\mathscr{E}(h)=\exp \left(\sum_{k=1}^{N} \int_{0}^{T} h_{k}(t) d w_{k}(t)-\frac{1}{2} \sum_{k=1}^{N} \int_{0}^{T}\left|h_{k}(t)\right|^{2} d t\right) .
$$

Полагая

$$
h_{k, i}=\int_{0}^{T} h_{k}(t) m_{i}(t) d t,
$$

мы можем переписать (4.16) в виде

$$
\mathscr{E}(h)=\exp \left(\sum_{i, k}\left(h_{k, i} \xi_{k i}-\frac{1}{2}\left|h_{k, i}\right|^{2}\right)\right)
$$

и получить

$$
\mathscr{E}(h)=\sum_{\alpha \in \mathscr{J}} \frac{h^{\alpha}}{\sqrt{\alpha !}} \xi_{\alpha}, \quad \text { где } \quad h^{\alpha}=\prod_{i, k} h_{k, i}^{\alpha_{i}^{k}} .
$$

Если $\theta_{h}(t)=\mathrm{E}(\theta(t) \mathscr{E}(h))$, то, объединяя (4.3), (4.6) и (4.17), получаем

$$
\theta_{h}(t)=\theta_{0}+\int_{0}^{t} \mathscr{A} \theta_{h}(s) d s+\int_{0}^{t} h_{k}(s) \mathscr{M}_{k} \theta_{h}(s) d s .
$$

Далее, пусть $\bar{\theta}_{\alpha}(t)$ - решение системы уравнений (4.3) с $\bar{m}_{i}$ вместо $m_{i}$. Из замечания 4.6 следует, что для любого $t \in[0, T]$ процесс $\bar{\theta}(t)=\sum_{\alpha} \bar{\theta}_{\alpha}(t) \bar{\xi}_{\alpha}(t)$ является элементом $L_{2}\left(\Omega ; L_{2}\left(\mathbb{R}^{d}\right)\right)$. Кроме того, полагая $\bar{\theta}_{h}(t)=\mathrm{E}(\bar{\theta}(t) \mathscr{E}(h))$ и замечая, что

$$
\mathscr{E}(h)=\exp \left(\sum_{i, k}\left(\bar{h}_{k, i} \bar{\xi}_{k i}-\frac{1}{2}\left|\bar{h}_{k, i}\right|^{2}\right)\right),
$$

где

$$
\bar{h}_{k, i}=\int_{0}^{T} h_{k}(t) \bar{m}_{i}(t) d t
$$

получаем

$$
\bar{\theta}_{h}(t)=\theta_{0}+\int_{0}^{t} \mathscr{A} \bar{\theta}_{h}(s) d s+\int_{0}^{t} h_{k}(s) \mathscr{M}_{k} \bar{\theta}_{h}(s) d s .
$$

Из единственности решения этого параболического уравнения следует равенство $\theta_{h}(t)=\bar{\theta}_{h}(t)$ в $L_{2}\left(\mathbb{R}^{d}\right)$ для любого $t \in[0, T]$ и всех конечных наборов $h_{1}, \ldots, h_{N}$ ограниченных измеримых функций на $(0, T)$. Так как соответствующее семейство элементов $\mathscr{E}(h)$ всюду плотно в $L_{2}(\mathscr{F} T)$, то для любого $t \in[0, T]$ имеем равенство $\theta(t)=\bar{\theta}(t)\left(\right.$ как элементов $\left.L_{2}\left(\Omega ; L_{2}\left(\mathbb{R}^{d}\right)\right)\right)$. Лемма 4.7 доказана.

Теперь мы можем установить предсказуемость процесса $\theta$ (шаг 2).

ЛЕмма 4.8. Прочесс $\theta$, определенный формулой (4.6), предсказуем. Если, кроме того, $\nu>0$, то процесс $\nabla \theta$ тоже предсказуем. 
ДокАЗАТЕЛЬСтво. Зафиксируем $t^{*} \in(0, T)$ и рассмотрим специальный базис $\bar{m}_{i}(t)$ в $L_{2}((0, T))$, удовлетворяющий условию, что носитель $\bar{m}_{i}$ содержится либо в $\left[0, t^{*}\right]$, либо в $\left[t^{*}, T\right]$. Обозначим через $\bar{\xi}_{\alpha}, \alpha \in \mathscr{J}$, соответствующий ортонормированный базис в $L_{2}\left(\mathscr{F}_{T}^{W}\right)$. Тогда из определения $\bar{\xi}_{\alpha}$ следует, что $\bar{\xi}_{\alpha}=\bar{\xi}_{\beta}\left(0, t^{*}\right) \bar{\xi}_{\gamma}\left(t^{*}, T\right)$, где $\bar{\xi}_{\beta}\left(0, t^{*}\right)$ (компонента, предшествуюшая $\left.t^{*}\right) \mathscr{F}_{t^{*}}$-измерима и $\bar{\xi}_{\gamma}\left(t^{*}, T\right)$ (компонента, следуюшая за $\left.t^{*}\right)$ независима от $\mathscr{F} t^{*}$. Соответственно, любой мультииндекс $\alpha \in \mathscr{J}$ будет представлен в виде $\alpha=(\beta, \gamma)$ для учета компоненты, предшествуюшей $t^{*}$, и компоненты, следующей за $t^{*}$. По лемме 4.7 ,

$$
\theta(t)=\sum_{\alpha \in \mathscr{J}} \bar{\theta}_{\alpha}(t) \bar{\xi}_{\alpha}=\sum_{\alpha \in \mathscr{J}} \bar{\theta}_{\beta, \gamma}(t) \bar{\xi}_{\beta}\left(0, t^{*}\right) \bar{\xi}_{\gamma}\left(t^{*}, T\right), \quad t \in[0, T],
$$

и коэффициенты $\bar{\theta}_{\alpha}$ удовлетворяют системе уравнений $(4.3)$ с $\bar{m}_{i}$ вместо $m_{i}$. Следовательно, при $t \leqslant t^{*}$ функция $\bar{m}_{i}$ входит в систему тогда и только тогда, когда носитель $\bar{m}_{i}$ содержится в $\left[0, t^{*}\right]$. Индукцией по $\beta$ получаем, что $\bar{\theta}_{\beta, \gamma}(t)=0$ при $t \in\left[0, t^{*}\right]$, если $|\gamma|>0$. С другой стороны, $\mathrm{E}\left(\bar{\xi}_{\gamma}\left(t^{*}, T\right) \mid \mathscr{F}_{t^{*}}^{W}\right)=\mathrm{E} \bar{\xi}_{\gamma}\left(t^{*}, T\right)=0$ для всех $\gamma$ с $|\gamma|>0$. В результате,

$$
\mathrm{E}\left(\theta\left(t^{*}\right) \mid \mathscr{F}_{t^{*}}^{W}\right)=\sum_{\alpha \in \mathscr{J}} \bar{\theta}_{\beta, 0} \bar{\xi}_{\beta}\left(0, t^{*}\right)=\theta\left(t^{*}\right)
$$

т.е. $\theta\left(t^{*}\right) \mathscr{F} W$-измерима для любого $t^{*} \in(0, T)$. То же рассуждение доказывает предсказуемость $\nabla \theta$ при $\nu>0$. Лемма 4.8 доказана.

Чтобы завершить доказательство теоремы 4.2 , остается установить равенства (4.7) и (4.9) (шаг 3). Как в доказательстве леммы 4.7, положим $\theta_{h}(t)=\mathrm{E}(\theta(t) \mathscr{E}(h))$, где $\mathscr{E}(h)$ определено формулой (4.16).

Если $\varphi \in C_{0}^{\infty}\left(\mathbb{R}^{d}\right)$, то из уравнения (4.3) следует, что

$$
\begin{aligned}
\left(\theta_{h}, \varphi\right)(t)= & (\theta(0), \varphi)+\int_{0}^{t}\left(\theta_{h}, \mathscr{A}^{*} \varphi\right)(s) d s \\
& +\sum_{\alpha \in \mathscr{J}} \frac{h^{\alpha}}{\alpha !} \sum_{i, k} \int_{0}^{t} \sqrt{\alpha_{i}^{k}} m_{i}(s)\left(\theta_{\alpha^{-}(i, k)}, \mathscr{M}_{k}^{*} \varphi\right)(s) d s
\end{aligned}
$$

где * означает переход к сопряженному оператору, $(\cdot, \cdot)$ - скалярное произведение в $\mathbb{R}^{n}$, а $h^{\alpha}$ определено в (4.17). Из свойств предсказуемости и интегрируемости процесса $\theta$ следует, что стохастический интеграл $I(t)=\int_{0}^{t}\left(\theta(s), \mathscr{M}_{k}^{*} \varphi\right) d w_{k}(s)$ корректно определен. Если $\xi_{\alpha}(t)=\mathrm{E}\left(\xi_{\alpha} \mid \mathscr{F}_{t}^{W}\right)$, то, согласно [13], имеем

$$
d \xi_{\alpha}(t)=\sum_{i, k} \sqrt{\alpha_{i}^{k}} \xi_{\alpha^{-}(i, k)}(t) m_{i}(t) d w_{k}(t)
$$

Ввиду $\mathscr{F}_{t}^{W}$-измеримости $I(t)$, получаем

$$
I_{\alpha}(t)=\mathrm{E}\left(I(t) \mathrm{E}\left(\xi_{\alpha} \mid \mathscr{F}_{t}^{W}\right)\right)=\mathrm{E}\left(I(t) \xi_{\alpha}(t)\right),
$$


а тогда из формулы Ито следует, что

$$
\mathrm{E}\left(I(t) \xi_{\alpha}(t)\right)=\int_{0}^{t} \sum_{i, k} \sqrt{\alpha_{i}^{k}} m_{i}(s)\left(\theta_{\alpha^{-}(i, k)}, \mathscr{M}_{k}^{*} \varphi\right)(s) d s .
$$

Из последнего соотношения, в сочетании с (4.17), следует, что

$$
\sum_{\alpha \in \mathscr{J}} \frac{z^{\alpha}}{\alpha !} \sum_{i, k} \int_{0}^{t} \sqrt{\alpha_{i}^{k}} m_{i}(s)\left(\theta_{\alpha^{-}(i, k)}, \mathscr{M}_{k}^{*} \varphi\right)(s) d s=\mathrm{E}\left(\mathscr{E}(h) \int_{0}^{t}\left(\theta(s), \mathscr{M}_{k}^{*} \varphi\right) d w_{k}(s)\right) .
$$

В результате получаем

$$
\begin{aligned}
\mathrm{E}(\mathscr{E}(h)(\theta, \varphi)(t))= & (\theta(0), \varphi)+\mathrm{E}\left(\mathscr{E}(h) \int_{0}^{t}\left(\theta, \mathscr{A}^{*} \varphi\right)(s) d s\right) \\
& +\mathrm{E}\left(\mathscr{E}(h) \int_{0}^{t}\left(\theta(s), \mathscr{M}_{k} \varphi\right) d w_{k}(s)\right) .
\end{aligned}
$$

Из равенства $(4.22)$ и из плотности семейства $\{\mathscr{E}(h)\}$ в $L_{2}(\mathscr{F} T)$, с учетом предположения $\mathbf{A 4}$ и равенства $\mathrm{E} \mathscr{E}(h)=1$, следует, что

$$
(\theta, \varphi)(t)=(\theta(0), \varphi)+\int_{0}^{t}\left(\theta, \mathscr{A}^{*} \varphi\right)(s) d s+\int_{0}^{t}\left(\theta, \mathscr{M}_{k}^{*} \varphi\right)(s) d w_{k}(s)
$$

Если $\nu=0$, то последнее равенство совпадает с (4.9). Если $\nu>0$, то из (4.8) видим, что соотношение (4.23) можно переписать в виде (4.7).

Это завершает доказательство теоремы 4.2.

Теорема Камерона-Мартина 3.2 и теорема 4.2 приводят к следующим простьм формулам для вычисления первых и вторых моментов решения пассивных скалярных уравнений (4.7) и (4.9).

СлЕДСТВИЕ 4.9. В предположсениях теоремь 4.2, для любых $s$ u $t$ u почтu всех $x$ и у имеем

$$
\mathrm{E} \theta(t, x)=\theta_{\alpha}(t, x) I_{|\alpha|=0}, \quad \mathrm{E} \theta(t, x) \theta(s, y)=\sum_{\alpha \in \mathscr{J}} \theta_{\alpha}(t, x) \theta_{\alpha}(s, y) .
$$

\section{5. Лагранжево представление решения}

Пусть $\widetilde{W}=\left(\widetilde{w}_{k}(s), k=1, \ldots, d, s \geqslant 0\right)$ - набор независимых стандартных винеровских процессов. Предположим, что $\widetilde{W}$ независим от $W=\left(w_{k}(s), k \geqslant 1, s \geqslant 0\right)$ и что $\nu \geqslant 0$. При некотором фиксированном $t, t<\infty$, рассмотрим следующее обратное уравнение Ито (см., например, [14]):

$$
\begin{gathered}
-d_{s} X_{t, x}^{i}(s)=\sqrt{2 \nu} \overleftarrow{d \widetilde{w}_{i}}(s)+\sigma_{k}^{i}\left(X_{t, x}(s)\right) \overleftarrow{d w_{k}}(s), \quad s \in[0, t), \\
X_{t, x}^{i}(t)=x
\end{gathered}
$$


В связи с нашим предположением, что $\operatorname{div}\left(\sigma_{k}\right)=0$, формы Ито и Стратоновича этого уравнения совпадают.

Так как каждая функция $\sigma_{k}^{i}(x)$ непрерьвна и, по предположению, $\sigma_{k}^{i}(x) \sigma_{k}^{j}(x)=$ $C^{i j}(0)$, отсюда следует, что для некоторого $\delta>0$ соотношение $\left(\sigma(x) \sigma^{*}(x) y, y\right) \geqslant \delta|y|^{2}$ вьполняется для всех $y \in \mathbb{R}^{d}$. Следовательно, уравнение (5.1) имеет мартингальное решение, т.е. можно найти стохастический базис $\mathbb{F}=\left(\Omega, \mathscr{F},\left\{\mathscr{F}_{t}\right\}_{t \geqslant 0}, \mathrm{P}\right)$, удовлетворяюший обычным требованиям, множество $B=(\widetilde{W}, W)=\left(\widetilde{w}_{k}(s), k=1, \ldots, d\right.$, $\left.w_{k}(s), k \geqslant 1, s \geqslant 0\right)$ независимых стандартных винеровских процессов, согласованных с $\left\{\mathscr{F}_{s}\right\}_{s} \geqslant 0$, и процесс $X_{t, x}(s)$ на $\mathbb{F}$ со значениями в $\mathbb{R}^{d}$ такие, что Р-почти наверное для всех $s<t$

$$
X_{t, x}(s)=x+\int_{s}^{t} \sigma\left(X_{t, x}(r)\right) \overleftarrow{d W}(r)+\sqrt{2 \nu}(\widetilde{W}(t)-\widetilde{W}(s))
$$

ЗАмЕЧАНИЕ 5.1. При $Y_{t, x}(s)=X_{t, x}(t-s)$ и $B_{t}(s)=B(t)-B(t-s)$ уравнение $(5.2)$ можно переписать следующим образом:

$$
Y_{t, x}(s)=x+\int_{0}^{s} \sigma\left(Y_{t, x}(r)\right) d W_{t}(r)+\sqrt{2 \nu} \widetilde{W}_{t}(t-s),
$$

где $W_{t}(r):=W(t)-W(t-r)$.

Заметим, что мартингальное (или слабое) решение уравнения (5.1) не обязательно $\mathscr{F}_{s}^{B}$-согласовано. Кроме того, нет никакой априорной гарантии того, что решение уравнения (5.1) может быть построено на заранее выбранном стохастическом базисе и для данного набора $B$ винеровских процессов. Грубо говоря, эти ограничения и составляют различие между мартингальным решением и сильным (в вероятностном смысле) решением, которое известно также как траекторное решение; см., например, [9] или [1].

С другой стороны, в теореме 4.2 мы доказали, что уравнение (2.5) имеет единственное $\mathscr{F}_{s}^{W}$-согласованное решение при любом $\nu \geqslant 0$ на любом стохастическом базисе и для любого набора $W$ независимых стандартных винеровских процессов на этом базисе. В частности, мы можем предположить - и будем предполагать, - что сушествует единственное траекторное решение $\theta=\theta(t, x)$ уравнения (2.5) на $\mathbb{F}$, управляемое $W$. Поэтому, если только $X_{t, x}(0)$ не является $\mathscr{F}_{t}^{W}$-измеримой, не следует ожидать классического лагранжева представления решения пассивного скалярного уравнения $\theta(t, x)=\theta_{0}\left(X_{t, x}(0)\right)$.

Однако справедливо следуюшее обобшение этой формулы.

Теорема 5.2. Для любого $T<\infty$ и для почти всех $x$ имеем

$$
\theta(T, x)=\mathrm{E}\left(\theta_{0}\left(X_{T, x}(0)\right) \mid \mathscr{F}_{T}^{W}\right) \quad(\mathrm{P}-\text { n. .. }) .
$$

ДокАЗАТЕльство. Как в доказательстве леммы 4.7 , определим величину $\mathscr{E}(h)$ как в (4.16) и функцию $\theta_{h}$ так, чтобы вьполнялось (4.18).

С другой стороны, по теореме Гирсанова

$$
\mathrm{E}\left(\mathscr{E}(h) \mathrm{E}\left(\theta_{0}\left(X_{T, x}(0)\right) \mid \mathscr{F}_{t}^{W}\right)\right)=\mathrm{E}\left(\mathscr{E}(h) \theta_{0}\left(X_{T, x}(0)\right)\right)=\mathrm{E}^{\prime}\left(\theta_{0}\left(X_{T, x}(0)\right)\right),
$$


где $\mathrm{E}^{\prime}$ - математическое ожидание относительно меры $d \mathrm{P}_{T}^{\prime}=\mathscr{E}(h) d \mathrm{P}_{T}$ и $\mathrm{P}_{T}$ - ограничение меры $\mathrm{P}$ на $\mathscr{F}_{T}$. Кроме того, $\mathrm{P}_{T}$-п.н. процесс $X_{T, x}(s)$ является мартингальньм решением уравнения

$$
\begin{aligned}
X_{T, x}(s)=x & +\int_{s}^{T} \sigma_{k}\left(X_{T, x}(r)\right) h_{k}(r) d r+\int_{s}^{T} \sigma\left(X_{T, x}(r)\right) \overleftarrow{d W^{\prime}}(r) \\
& +\sqrt{2 \nu}\left(\widetilde{W^{\prime}}(T)-\widetilde{W^{\prime}}(s)\right), \quad s \leqslant T .
\end{aligned}
$$

По формуле Фейнмана-Каца, функция $\psi_{h}(s):=\mathrm{E}^{\prime}\left(\theta_{0}\left(X_{t, x}(s)\right)\right)$ тоже является решением уравнения (4.18). Из единственности решения уравнения (4.18) следует, что $\mathrm{E}\left(\mathscr{E}(h) \mathrm{E}\left(\theta_{0}\left(X_{T, x}(0)\right) \mid \mathscr{F}_{T}^{W}\right)\right)=\mathrm{E}(\mathscr{E}(h) \theta(T, x))$ для любого конечного набора $h$ ограниченных измеримых функций на $[0, T]$. Так как набор всех таких $\mathscr{E}(h)$ всюду плотен в $L_{2}\left(\Omega, \mathscr{F}_{t}^{W}\right)$, то Р-п.н. имеем $\theta(T, \cdot)=\mathrm{E}\left(\theta_{0}\left(X_{t}, \cdot(0)\right) \mid \mathscr{F}_{T}^{W}\right)$ (как равенство элементов $\left.L_{2}\left(\Omega ; \mathbb{R}^{d}\right)\right)$. Это завершает доказательство теоремы 5.2 .

ТеОРема 5.3. Энергетическое равенство $\mathrm{E}\|\theta\|_{L_{2}\left(\mathbb{R}^{d}\right)}^{2}(t)=\left\|\theta_{0}\right\|_{L_{2}\left(\mathbb{R}^{d}\right)}^{2}$ выполняется тогда и только тогда, когда для почти всех $x$

$$
\mathrm{E}\left(\theta_{0}\left(X_{t, x}(0)\right) \mid \mathscr{F}_{t}^{W}\right)=\theta_{0}\left(X_{t, x}(0)\right) \quad(\mathrm{P}-\text { n.н. }) .
$$

ДокАЗАТЕльство. Предположим, что вьполняется условие (5.3). Плотность вероятности перехода процесса $X$ однородна, т.е. имеет вид $p(t, x-y)$. Тогда

$$
\begin{aligned}
\mathrm{E}\|\theta\|_{L_{2}\left(\mathbb{R}^{d}\right)}^{2}(t) & =\int_{\mathbb{R}^{d}} \mathrm{E}\left|\theta_{0}\left(X_{t, x}(0)\right)\right|^{2} d x \\
& =\int_{\mathbb{R}^{d}} \int_{\mathbb{R}^{d}}\left|\theta_{0}(y)\right|^{2} p(t, x-y) d x d y=\left\|\theta_{0}\right\|_{L_{2}\left(\mathbb{R}^{d}\right)}^{2} .
\end{aligned}
$$

Предположим теперь, что для любого $t$ существует множество $\Gamma \subseteq \mathbb{R}^{d}$ положительной лебеговой меры такое, что $\mathrm{E}\left(\theta_{0}\left(X_{t, x}(0)\right) \mid \mathscr{F}_{t}^{W}\right) \neq \theta_{0}\left(X_{t, x}(0)\right)$. По неравенству Минковского имеем $\mathrm{E}\left|\mathrm{E}\left(\theta_{0}\left(X_{t, x}(0)\right) \mid \mathscr{F}_{t}^{W}\right)\right|^{2} \leqslant \mathrm{E}\left|\theta_{0}\left(X_{t, x}(0)\right)\right|^{2}$, и равенство выполняется, только если $\mathrm{E}\left(\theta_{0}\left(X_{t, x}(0)\right) \mid \mathscr{F}_{t}^{W}\right)=\theta_{0}\left(X_{t, x}(0)\right)$. Поэтому

$$
\begin{aligned}
\mathrm{E}\|\theta\|_{L_{2}\left(\mathbb{R}^{d}\right)}^{2}(t) & =\mathrm{E} \int_{\mathbb{R}^{d}}\left|\mathrm{E}\left(\theta_{0}\left(X_{t, x}(0)\right) \mid \mathscr{F}_{t}^{W}\right)\right|^{2} d x<\int_{\mathbb{R}^{d}} \mathrm{E}\left|\theta_{0}\left(X_{t, x}(0)\right)\right|^{2} d x \\
& =\int_{\mathbb{R}^{d}} \int_{\mathbb{R}^{d}} \mathrm{E}\left|\theta_{0}(y)\right|^{2} p(t, x-y) d y d x=\left\|\theta_{0}\right\|_{L_{2}\left(\mathbb{R}^{d}\right)}^{2} .
\end{aligned}
$$

Это доказьвает теорему 5.3.

ЗАмЕчАнИЕ 5.4. Очевидно, что во всех интересных сценариях условие (5.3) не выполняется, если $\nu>0$. Если $\nu=0$, то условие (5.3) эквивалентно предположению, что $X_{t, x}(s)$ является сильным решением уравнения (5.1).

Теоремы 4.2 и 5.2 приводят к следуюшей оценке нормы решения уравнения (2.5).

ТеОРема 5.5. Если $\theta_{0} \in L_{p}\left(\mathbb{R}^{d}\right), 2 \leqslant p<\infty$, то для любого $t \geqslant 0$ решение $\theta=\theta(t, x)$ уравнения (2.5) удовлетворяет неравенству

$$
\mathrm{E}\|\theta\|_{L_{p}\left(\mathbb{R}^{d}\right)}^{p}(t) \leqslant\left\|\theta_{0}\right\|_{L_{p}\left(\mathbb{R}^{d}\right)}^{p} .
$$


ДоказАТельство. Обозначим через $S_{t}: \theta_{0}(\cdot) \mapsto \theta(t, \cdot), t>0$, оператор решения для уравнения (2.5). По теореме $4.2, S_{t}$ является ограниченным линейным оператором из $L_{2}\left(\mathbb{R}^{d}\right)$ в $L_{2}\left(\Omega \times \mathbb{R}^{d}\right)$ и

$$
\left\|S_{t} \theta_{0}\right\|_{L_{2}\left(\Omega \times \mathbb{R}^{d}\right)} \leqslant\left\|\theta_{0}\right\|_{L_{2}\left(\mathbb{R}^{d}\right)} .
$$

По теореме $5.2, S_{t}$ есть ограниченньй линейный оператор из $L_{\infty}\left(\mathbb{R}^{d}\right)$ в $L_{\infty}\left(\Omega \times \mathbb{R}^{d}\right)$ и

$$
\left\|S_{t} \theta_{0}\right\|_{L_{\infty}\left(\Omega \times \mathbb{R}^{d}\right)} \leqslant\left\|\theta_{0}\right\|_{L_{\infty}\left(\mathbb{R}^{d}\right)}
$$

Теперь неравенство (5.4) следует из теоремы Рисса о выпуклости (см., например, [3; теорема 4.1.7]).

ЗАмечАниЕ 5.6. Можно было бы показать, что оператор, сопряженный к оператору решения в теореме 3.2 из [11], является обобшенным решением уравнения (4.7) с $\nu \geqslant 0$. Однако, поскольку мы рассматриваем гораздо более специализированную ситуацию, то доказательство существования, предложенное в настоящей статье, оказывается проше. Главные новые элементы нашей статьи включают:

(1) единственность сильного решения невьнужденного несжимаемого (unforced) пассивного скалярного уравнения для подходящего класса начальных условий,

(2) $L_{p}$-регулярность решений (в терминах $L_{p}$-регулярности начального условия),

(3) процедуру вычисления решения и моментов пассивного скалярного уравнения через стохастические коэффициенты $\Phi$ урье $\theta_{\alpha}$.

\section{СПИСОК ЛИТЕРАТУРЫ}

[1] С. В. Анулова, А. Ю. Веретенников, Н. В. Крылов, Р. Ш. Липцер, А. Н. Ширяев. Стохастическое исчисление // Итоги науки и техники. Фундам. напр. Т. 45. М.: ВИНИТИ, 1989. С. 5-253.

[2] P. Baxendale, T. E. Harris. Isotropic stochastic flows // Ann. Probab. 1986. V. 14. № 4. P. 1155-1179.

[3] C. Bennett, R. Sharpley. Interpolation of Operators. Boston: Academic Press, 1988. (Pure Appl. Math. V. 129.)

[4] R.H. Cameron, W.T. Martin. The orthogonal development of nonlinear functionals in series of Fourier-Hermite functionals // Ann. of Math. (2). 1947. V. 48. № 2. P. 385-392.

[5] W. E, E. Vanden Eijden. Generalized flows, intrinsic stochasticity, and turbulent transport // Proc. Natl. Acad. Sci. USA. 2000. V. 97. № 15. P. 8200-8205.

[7] K. Gawȩdzki, M. Vergassola. Phase transition in the passive scalar advection // Phys. D. 2000. V. 138. №1-2. P. 63-90.

[6] K. Gawedzki, A. Kupiainen. Universality in turbulence: an exactly solvable model // Lecture Notes in Phys. 1996. V. 469. P. 71-105.

[8] T. Hida, H.-H. Kuo, J. Potthoff, L. Streit. White Noise. An Infinite-dimensional Calculus. Dordrecht: Kluwer, 1993.

[9] Ш. Ватанабэ, Н. Икэда. Стохастические дифференциальные уравнения и диффузионные процессы. М.: Наука, 1985.

[10] R.H. Kraichnan. Small-scale structure of a scalar field convected by turbulence // Phys. Fluids. 1968. V. 11. P. 945-963.

[11] Y. Le Jan, O. Raimond. Integration of Brownian vector fields // Ann. Probab. 2002. V. 30. № 2. P. 826-873. 
[12] S. Lototsky, R. Mikulevicius, B. L. Rozovskii. Nonlinear filtering revisited: a spectral approach // SIAM J. Control Optim. 1997. V. 35. № 2. P. 435-461.

[13] R. Mikulevicius, B. L. Rozovskii. Linear parabolic stochastic PDEs and Wiener chaos // SIAM J. Math. Anal. 1998. V. 29. № 2. P. 452-480.

[14] Б. Л. Розовский. Эволюционные стохастические системы. М.: Наука, 1983.

University of Southern California, Los Angeles

E-mail: lototsky@math.usc.edu, rozovski@math.usc.edu
Поступила в редакцию 20.06 .2003 